\title{
Manutenção de Intervenções de Atividade Física: premissas para eficácia individual
}

\section{Maintenance of Physical Activity Interventions: premises of efficacy individual}

\section{Mantenimiento de Intervenciones de Actividad Física: premisas para la eficacia individual}

\section{Recebido: 10/06/2013 \\ Aprovado: 21/02/2014}

Thomas Richard Wójcicki1

Edward McAuley²

Esta é uma revisão que tem como objetivo apresentar investigações sobre atividade física examinando o aspecto Manutenção do modelo RE-AIM. Os estudos citados realizaram-se com adultos de meia-idade e idosos. Considerou-se manutenção ao nível individual, tipicamente representada, pelo grau em que as alterações iniciais no comportamento do participante são mantidas ao longo do tempo. Na maioria dos casos, isso reflete seis meses ou mais pós-intervenção. Em particular, foi analisada em que medida a auto-eficácia está relacionada com a manutenção da atividade física. Este trabalho sugere que as percepções de eficácia pessoal são importantes não só para adoção inicial deste comportamento de saúde, mas também para a manutenção por períodos maiores que cinco anos. É importante ressaltar que esses efeitos são sustentados quando o comportamento passado é controlado. Como a auto-eficácia é modificável, é possível aos intervencionistas traçarem estratégias para melhorar a eficácia maximizando assim a manutenção.

Descritores: Exercício; Atividade motora; Autoeficácia; Estudos de intervenção; Comportamentos saudáveis.

This is a review aim to present of a physical activity research program examining the Maintenance aspect of the RE-AIM model. The studs considered with middle-aged and older adults. It is consider maintenance at the individual level typically represented by the degree to which initial changes in participant behavior are sustained over time. In most cases, this reflects six months or more post intervention. In particular it review the extent to which self-efficacy is implicated in the maintenance of physical activity. This work suggests that perceptions of personal efficacy are important for initial adoption of this health behavior but also for maintenance for periods as much as five years. Importantly, these effects are sustained when past behavior is controlled. As self-efficacy is modifiable, it should be possible for interventionists to design strategies to enhance efficacy thereby maximizing maintenance.

Descriptors: Exercise; Motor activity; Self efficacy; Intervention studies; Health behavior.

Esta es una revisión que tiene como objetivo presentar investigaciones sobre actividad física examinando el ítem Mantenimiento del modelo RE-AIM. Los estudios citados se realizaron con adultos de media edad y ancianos. Se consideró mantenimiento al nivel individual, típicamente representado, por el grado en que las alteraciones iníciales en el comportamiento del participante son mantenidas a lo largo del tiempo. En la mayoría de los casos, eso refleja seis meses o mas pos-intervención. En particular, fue analizada en qué medida la auto-eficacia está relacionada con el mantenimiento de la actividad física. Este trabajo sugiere que las percepciones de eficacia personal son importantes no solo para la adopción inicial de este comportamiento de salud, sino también para el mantenimiento por períodos mayores a cinco años. Es importante resaltar que esos efectos son sustentados cuando el comportamiento pasado ha sido controlado. Como la auto-eficacia es modificable, es posible para los intervencionistas trazar estrategias para mejorar la eficacia maximizando así el mantenimiento.

Descriptores: Ejercicio; Actividad motora; Auto-efectividad; Estudios de intervención; Comportamientos saludables.

\footnotetext{
1 Educador Físico. Doutorando em Cinesiologia. Departamento de Cinesiologia e Saúde Comunitária da Universidade de Illinois UrbanaChampaign, USA. wojcicki@illinois.edu

2 Educador Físico. Mestre em Educação com ênfase em Psicologia do Desporto. Doutor em Esporte e Psicologia do Exercício. Professor do Curso de Mestrado e Doutorado em Cinesiologia. Departamento de Cinesiologia e Saúde Comunitária da Universidade de Illinois UrbanaChampaign, USA. emcauley@illinois.edu
} 


\section{INTRODUÇÃO}

Dados recentes sugerem que indivíduos que aderem à atividade física reduzem significativamente a mortalidade se comparados com aqueles que não aderem ${ }^{1}$. No entanto, a adesão a regimes de saúde, tais como o exercício regular, controle de peso e uso de medicamentos a longo prazo são notoriamente difíceis.

Manutenção, no âmbito do modelo RE-AIM, manifesta-se tanto no nível individual como no nível organizacional. Neste último, a manutenção é determinada pela avaliação da medida em que um programa ou política é institucionalizado ou torna-se parte das práticas organizacionais. A manutenção ao nível individual é representada pelo grau em que as alterações iniciais no comportamento do participante são mantidas ao longo do tempo, tipicamente, isto é refletido em seis meses ou mais após intervenção.

Este trabalho é fundamentado na teoria social cognitiva ${ }^{2}$ e, em particular, no papel desempenhado pela autoeficácia na efetivação da manutenção de atividade física. A autoeficácia é considerada o "agente ativo" na teoria social cognitiva e reflete as crenças a respeito das capacidades de um indivíduo para realizar com sucesso um curso de ação ${ }^{3}$.

A autoeficácia pode ser considerada como uma forma específica de autoconfiança para cada situação. Como as expectativas da autoeficácia podem ser sujeitas tanto a influências externas quanto internas, tornam-se alvos ideais para a manipulação $0^{4} \mathrm{e}$ intervenção ${ }^{5}$.

As principais fontes de informação sobre a eficácia incluem conquistas no passado (experiências diretas), a persuasão social, a modelagem social e a interpretação de estados físicos e emocionais ${ }^{2}$.

A eficácia pode exercer influência sobre as atividades individuais escolhidas por cada pessoa para prosseguir, o grau de esforço que eles despendem em busca de seus objetivos e os níveis de persistência demonstrada em face de retrocessos, fracassos e dificuldades. Claramente, a escolha, esforço e persistência são importantes elementos para o êxito na adoção e manutenção de comportamentos de atividade física.

A autoeficácia parece ter um correlato natural a esse comportamento de saúde complexo e, de fato, tem sido um dos correlatos mais frequentemente apontado como importante para atividade física e os seus resultados ${ }^{6}$. Subsequentemente, acredita-se que um enfoque neste correlato do comportamento da atividade física é importante para a consideração da manutenção no nível individual como parte integrante do modelo RE-AIM. O objetivo desta revisão é apresentar investigações sobre a associação da autoeficácia com a Manutenção da atividade física.

\section{MÉTODO}

Esta é uma breve revisão sobre pesquisas realizadas em um Centro de Pesquisas norte americano voltadas à atividade física e à autoeficácia, com destaque para a demonstração do quesito Manutenção do modelo RE-AIM.

Os dados foram sintetizados a partir de vários estudos prospectivos e experimentos de exercício físico realizados no Laboratório de Psicologia do Exercício da Universidade de Illinóis, EUA. Como tal, é relatada a associação da autoeficácia com a manutenção da atividade física por períodos que variam de quatro meses a cinco anos pós-intervenção.

Não houve a preocupação de sistematização no levantamento das referidas publicações, já que o foco era o quesito Manutenção do modelo RE-AIM para avaliação da atividade física em adultos e idosos, considerando ainda ser o referido Centro de Pesquisa referência tanto em atividade física como em testagem do modelo RE-AIM, não havendo tantos centros que combinem ao mesmo tempo a atividade Física e a testagem do modelo em questão, o que justifica a importância desta comunicação científica. 


\section{RESULTADOS}

São apresentadas 11 publicações referentes a pesquisas desenvolvidas no Laboratório de Psicologia do Exercício da Universidade de Illinóis, Estados Unidos da América, com foco na associação da autoeficácia com a atividade física em adultos e idosos, abordando a dimensão Manutenção do Modelo RE-AIM.

Dentre as publicações, uma é da década de 1980, seis da década de 1990, três da década de 2000 e uma da década de 2010. Das 11 publicações 10 são artigos e estão distribuídos em seis periódicos diferentes, três deles ligados a Comportamentos em Saúde, um à Saúde do Idoso, um à Medicina Preventiva e outro direcionado à Epidemiologia. Uma delas é referente a livro.

\section{DISCUSSÃO}

Em exame sistemático do papel desempenhado pela autoeficácia na manutenção da atividade física em adultos de meia-idade e mais velhos ao longo das últimas duas décadas, mostram verificações importantes. Em um estudo7, a autoeficácia, a partir de dados iniciais de um programa de cinco meses de duração para adultos de meia-idade $(N=103)$ com baixo nível de atividade, foi preditivo de adoção durante os três primeiros meses, com indivíduos mais eficazes demonstrando maior frequência de participação.

Na tentativa de se considerar como a autoeficácia influencia a manutenção, uma avaliação de acompanhamento foi realizada nove meses após a coleta inicial de dados ${ }^{8}$. Dos 103 participantes originais, 82 indivíduos $\quad(79,61 \%)$ completaram as avaliações neste momento. A autoeficácia para continuar se exercitando foi avaliada no final do programa de cinco meses para prever a participação em exercícios físicos no acompanhamento (avaliada através de entrevistas por telefone). A capacidade aeróbica, nível de exercício físico anterior, e a intensidade do exercício durante o programa também foram incluídos como preditores de atividade futura, no referido estudo. Todas as variáveis preditoras foram correlacionadas com o comportamento de exercício físico ao nível bivariado, mas apenas a autoeficácia emergiu como um preditor significativo da participação de exercícios ao longo de quatro meses de seguimento.

Dada a maleabilidade de autoeficácia como um constructo, tais achados podem sugerir que o foco na autoeficácia como um componente chave das intervenções de atividade física pode ser rentável para a manutenção e reforçar ainda mais a mesma no nível individual.

Com base nestes resultados encorajadores, McAuley et $\mathrm{al}^{5}$ conduziram um estudo randomizado controlado baseado em eficácia para analisar engajamento em atividades físicas em diversas etapas do processo de se exercitar (ou seja, adoção, adaptação e manutenção). Adultos sedentários de meia idade $(\mathrm{N}=114)$ foram recrutados para participar do programa e foram aleatoriamente designados para $o$ grupo de intervenção ou o grupo controle. Ambos os grupos participaram de um programa de exercícios progressivos realizados três dias por semana por cinco meses, sendo que cada sessão de uma hora consistia de aquecimento, um período de atividade aeróbica (caminhada) e um desaquecimento.

Com base nas proposições da teoria da autoeficácia ${ }^{9}$, a adesão em intervenções incorpora as quatro fontes primárias de informação com base na eficácia (experiências diretas, modelagem social, persuasão social e interpretação de estados fisiológicos) provenientes dos dados iniciais até a finalização do programa aos cinco meses. 0 grupo controle recebeu informações relacionadas à saúde ao longo do programa. Os resultados revelaram efeitos de tratamento significativos para o grupo de intervenção com base em eficácia, em comparação com o grupo controle. A cada mês, o grupo de intervenção exercitouse com mais frequência (ds=0,40-0,59), passou mais tempo envolvido em exercício 
(ds=0,31-0,55), e caminhou distâncias maiores (ds=0,23-0,52) do que o grupo controle. A auto-eficácia foi um preditor significativo de comportamento de exercício físico nas fases iniciais e intermediárias do programa de exercício, mas não no final do programa.

Este resultado está de acordo com outras pesquisas ${ }^{7,10}$ que indicam que a autoeficácia desempenha um papel mais importante na fase inicial de mudanças no comportamento de exercício físico (ou seja, adoção e adaptação); todavia, como o comportamento torna-se mais rotineiro e, possivelmente, menos exigente ao longo do tempo, o papel da autoeficácia como um preditor pode começar a diminuir.

Os estudos apresentados fornecem evidências que sugerem que os ensaios de eficácia são mais sensíveis durante as fases iniciais do processo de exercício fisico ${ }^{5}$ e que a autoeficácia, ao final de um programa de exercício estruturado, é um preditor de exercício significativo ${ }^{8}$. No entanto, estes resultados são específicos a um programa de exercício baseado em caminhada limitando a generalização que, pelo menos, em certa medida, como outros modos de exercício pode produzir resultados conflitantes e desfechos alternativos.

A fim de solucionar isso, McAuley et al $^{11}$ recrutaram 174 adultos idosos sedentários para participar de um ensaio clínico randomizado de seis meses, examinando os efeitos de duas modalidades de exercício (caminhada versus alongamento /musculação) sobre mudanças na autoeficácia de exercício, bem como a autoeficácia para superar barreiras. Aulas de exercícios foram dadas para ambas as condições, com duração de uma hora, três vezes por semana. A intervenção de caminhada foi especificamente concebida para influenciar a aptidão aeróbica, enquanto a intervenção, o alongamento e a tonificação eram focados em flexibilidade e força. Ao final do programa, avaliações de seguimento para determinar preditores e padrões de manutenção de longo prazo foram realizadas entre seis a 18 meses $^{12}$.

A adesão ao programa de seis meses foi de $88,0 \%$ e não houve diferenças entre os diferentes grupos de exercício. No seguimento, $80,0 \%$ da amostra original estavam dispostos a participar e, análises preliminares indicaram que os homens foram significativamente mais ativos, tanto em seis e 18 meses de seguimento.

É importante ressaltar que os participantes tinham mantido seu nível de atividade física, como medido pela Escala de Atividade Física para Idosos (PASE13). Modelos de equações estruturais testaram vários modelos concorrentes de predição de atividade física. 0 final e melhor modelo ajustado revelou que a maior frequência de exercício durante os seis meses do programa, o maior efeito positivo em relação a ser ativo, e o maior apoio social foram associados com uma maior autoeficácia.

Por sua vez, a autoeficácia teve um efeito direto significativo na manutenção do exercício físico em seis a 18 meses de seguimento. Assim, os indivíduos que são mais eficazes em exercitar-se no final do programa de seis meses também são os mais propensos a manter o comportamento no futuro próximo. Particularmente importante é o fato de que a autoeficácia é um preditor de manutenção da atividade física independente do comportamento do exercício no passado.

Esta coorte foi acompanhada para a determinação das contribuições de autoeficácia na previsão da manutenção da atividade física em longo prazo através da coleta de dados de atividade física, cinco anos após o término do programa ${ }^{14}$. Neste período de avaliação, $72,4 \%(n=126)$ da amostra original permaneceram no estudo.

Houve um declínio de 21,0\% na atividade física, avaliada pelo PASE, desde os 18 meses de seguimento, com níveis médios de atividade física decaindo abaixo do patamar inicial. No entanto, as maiores quedas foram no grupo de alongamento/ musculação, enquanto o grupo de caminhada 
relatou níveis de atividade após cinco anos acima do patamar inicial. Análises subsequentes utilizando modelagem de covariância revelou que os valores do PASE em 18 meses de seguimento foram os mais fortes preditores dos valores do PASE em cinco anos; entretanto, uma maior eficácia no fim do programa e maior afeto positivo continuaram a ter um efeito significativo sobre o nível de atividade física em cinco anos independente do comportamento anterior.

Em um estudo recente ${ }^{15}$, relatou-se dados de uma intervenção de 12 meses de atividade física para idosos em que os participantes $(N=179)$ foram randomizados para um grupo de caminhada ou um grupo de flexibilidade, tonificação e equilíbrio. Medidas de função executiva e uso de estratégia de auto-regulação foram avaliadas no início do estudo, enquanto a autoeficácia foi avaliada a cada três semanas no programa. A manutenção da atividade física foi medida pelo comparecimento aos 11 meses subsequentes no programa.

0 percentual de comparecimento foi de $68,2 \%$ no total e não diferiu por grupo. Análises de percurso indicaram que o uso de estratégias de auto-regulação e função cognitiva (executiva) predisseram significativamente a manutenção, porém indiretamente, através da autoeficácia. Tais achados reforçam a importância da função executiva (capacidade de planejar, multitarefa, inibir respostas) e autoeficácia na habilidade pessoal de se auto-regular comportamentos de saúde complexos.

A partir dos resultados sugeriu-se que a avaliação da função cognitiva antes de intervenções pode ser útil na identificação daqueles que podem ter dificuldade em manter o comportamento ao longo do tempo. Tal conhecimento pode ser usado no desenvolvimento de estratégias para promover a adoção e manutenção de exercício físico, com vistas à prevenção de desistências.

A Manutenção em longo prazo no nível individual é uma importante questão de saúde coletiva; mas a manutenção do comportamento da atividade física em estudos de eficácia raramente é avaliada ${ }^{16}$. Isso é lamentável pois a manutenção bemsucedida de comportamentos de saúde direcionados pode auxiliar na determinação do valor e eficácia de diversas intervenções comportamentais.

\section{CONCLUSÃO}

Os estudos destacados nesta mini-revisão são de um programa de pesquisa para compreender a dimensão Manutenção do modelo RE-AIM sob uma perspectiva social cognitiva. Os resultados ressaltam a importância e a influência da autoeficácia na gestão comportamental e manutenção em longo prazo.

Os estudos revisados demonstraram consistentemente que a autoeficácia pode ter efeitos significativos em curto e longo prazo sobre a manutenção independente de atividade física (pós-intervenção). $\quad \mathrm{Na}$ medida em que esses resultados são generalizáveis, todavia, ainda não foram determinadas uma vez que as amostras dos estudos relatados aqui foram autoselecionadas e relativamente homogêneas em termos de fatores demográficos, e participaram de exercícios em ambientes altamente organizados e supervisionados, bem como ligados a um único Centro de Pesquisa.

Os estudos apresentados indicam que os indivíduos com níveis mais altos de atividades físicas relacionadas com a autoeficácia no final do programa são mais propensos a se engajar e manter o comportamento em longo prazo.

A natureza progressiva da autoeficácia em relação à adesão e manutenção é promissora, já que este constructo pode ser facilmente direcionado e modificado durante todo o curso de uma intervenção de exercícios físicos.

Os ambientes de exercícios físicos que alvejam as fontes de autoeficácia devem ser encorajados, não só para maximizar a aderência ao programa em si, mas para 
influenciar a manutenção eventual do comportamento no futuro.

Cabe acrescentar que os pesquisadores devem considerar a possibilidade de estratégias para reforçar a autoeficácia nas fases posteriores das intervenções, assim como realizar pelo menos uma avaliação de seis meses de acompanhamento para analisar as mudanças no comportamento de interesse, bem como a eficácia global do programa.

Em última instância, se os efeitos de uma intervenção comportamental não são sustentáveis individualmente, então não é provável que tenha um grande impacto na atenção primária à saúde.

\section{REFERÊNCIAS}

1. Schoenborn CA, Stommel M. Adherence to the 2008 adults physical activity guidelines and mortality risk. Am J Prev Med. 2011; 40(5):514-21.

2. Bandura A. Self-Efficacy: the exercise of control. New York: Freeman; 1997.

3. Bandura A. Social learning theory. Englewood Cliffs, NJ: Prentice-Hall; 1977.

4. McAuley E, Talbot HM, Martinez S. Manipulating self-efficacy in the exercise environment in women: influences on affective responses. Health Psychol. 1999; 18(3):288-94.

5. McAuley E, Courneya KS, Rudolph DL, Lox CL. Enhancing exercise adherence in middleaged males and females. Prev Med. 1994; 23(4):498-06.

6. McAuley E, Blissmer B. Social cognitive determinants and consequences of physical activity. Exerc Sport Sci Rev. 2000; 28(2):858.

7. McAuley E. The role of efficacy cognitions in the prediction of exercise behavior in middle-aged adults. J Behav Med. 1992; 15(1):65-88.

8. McAuley E. Self-efficacy and the maintenance of exercise participation in older adults. J Behav Med. 1993; 16(1):10313.
9. Bandura A. Social foundations of thought and action: a social cognitive theory. Englewood Cliffs, NJ: Prentice-Hall; 1986. $544 \mathrm{p}$.

10. Bandura A. Human agency in social cognitive theory. Am Psychol. 1989; 44(9):1175-84.

11. McAuley E, Katula J, Mihalko SL, Blissmer B, Duncan TE, Pena M, et al. Mode of physical activity and self-efficacy in older adults: a latent growth curve analysis. J Gerontol B Psychol Sci Soc Sci. 1999; 54(5):P283-92.

12. McAuley E, Jerome GJ, Elavsky S, Marquez DX, Ramsey SN. Predicting longterm maintenance of physical activity in older adults. Prev Med. 2003; 37(2):110-8.

13. Washburn RA, Smith KW, Jette AM, Janney CA. The physical activity scale for the elderly (PASE): development and evaluation. J Clin Epidemiol. 1993; 46(2):153-62.

14. McAuley E, Morris KS, Motl RW, Hu L, Konopack JF, Elavsky S. Long-term follow-up of physical activity behavior in older adults. Health Psychol. 2007; 26(3):375-80.

15. McAuley E, Mailey EL, Mullen SP, Szabo AN, Wójcicki TR, White SM, et al. Growth trajectories of exercise self-efficacy in older adults: influence of measures and initial status. Health Psychol. 2011; 30(1):75-83.

16. Marcus BH, Dubbert PM, Forsyth LH, McKenzie TL, Stone EJ, Dunn AL, et al. Physical activity behavior change: issues in adoption and maintenance. Health Psychol. 2000; 19 (1 Suppl):32-41.

\section{CONTRIBUIÇÕES}

Thomas Richard Wójcicki contribuiu no desenho, na análise de dados, na interpretação e discussão dos resultados, e redação do artigo.

Edward McAuley contribuiu no desenho, na análise de dados, na interpretação e discussão dos resultados, e redação do artigo.

\section{AGRADECIMENTOS}

Agradecimentos ao National Institute on Aging, EUA pelo financiamento do referido estudo (Grant \#s AG 025667; AG 020118; AG 012113; 07907). 